\title{
Original
}

\section{Change in the Magnetic Resonance Imaging Signal of the Mandibular Condyle Due to Bisphosphonate-Related Osteonecrosis of the Jaw}

\author{
Naohisa Hirahara, Hirotaka Muraoka, Marie Noda, Teruaki Muramatsu, \\ Satoshi Tokunaga and Takashi Kaneda
}

Department of Radiology, Nihon University School of Dentistry at Matsudo, Chiba, Japan

(Accepted for publication, December 16, 2016)

\begin{abstract}
Marx was the first to report osteonecrosis of the jaw due to administration of BPs in 2003. Bisphosphonate-related osteonecrosis of the jaw (BRONJ) is the result of an adverse drug reaction. Moreover, many studies worldwide have reported an association between a range of serious dental diseases and the use of bisphosphonates (BPs). Few studies, however, have evaluated BRONJ based on an abnormal signal detected by MRI in the bone marrow of the mandibular condyle. The aim of this study was to assess changes in the magnetic resonance imaging (MRI) signal from the mandibular condyle that could be due to BRONJ. In particular, we focused on the presence of an abnormal MRI signal emanating from mandibular condyle bone marrow. Twenty-eight patients (11 men, 17 women; 56 temporomandibular joints) with BRONJ were evaluated for jaw pain. The patients, whose mean age was $72.9 \pm 9.4$ years (range 48 - 88 years), underwent MRI examination of the jawbone at our hospital from August 2006 to December 2015 and were included in the study. Overall, $80.0 \%$ of the patients diagnosed with BRONJ exhibited an abnormal bone marrow signal in the mandibular condyle on the same side of the face that suffered jaw pain. This abnormal signal was present significantly more frequently on the side of the face with the jaw symptoms than on the side without symptoms. Patients with BRONJ displayed an abnormal MRI signal in the mandibular condyle on the side of the face with jaw symptoms, suggesting that MRI findings could be useful clinically for detecting BRONJ in the mandibular condyle.
\end{abstract}

Key words: Magnetic resonance imaging signal, Mandibular condyle, Bisphosphonate-related osteonecrosis of the jaw, Bone marrow signal

\section{Introduction}

Bisphosphonate-related osteonecrosis of the jaw (BRONJ) is the result of an adverse drug reaction. It manifests as progressive destruction and death of bone that affects the mandible or maxilla of patients treated with nitrogen-containing bisphosphonates (BPs) in the absence of previous radiation treatment ${ }^{1)}$. Currently, BPs are used in all cases in which it is deemed necessary to prevent bone resorption ${ }^{2)}$. BPs such as alendronate, risedronate, ibandronate, and clodronate are used to treat several metabolic and oncologic pathologies that promote destruction of the skeletal system. They are administered by intravenous injection to treat metastatic osteolytic problems caused by several conditions, including multiple myeloma, bone metastasis from solid tumors with or without hypercalcemia (e.g., breast cancer, prostate cancer, cancer of the kidney or lung, Paget's disease) $)^{3)}$. The reported incidence of BRONJ is significantly higher with the intravenous preparations zoledronic acid and pamidronate, whereas the risk

Correspondence to: Dr. Naohisa Hirahara, Department of Radiology, Nihon University School of Dentistry at Matsudo, 2-870-1 SakaechoNishi, Matsudo, Chiba, 271-8587 Japan; Tel.: +81-47-360-9419; Fax: +81-47-364-6295; E-mail: mana13014@g.nihon-u.ac.jp appears to be minimal for patients receiving oral BPs. The mandible is more commonly affected than the maxilla (2:1 ratio), and $60 \%$ of cases are preceded by a dental surgical procedure. The signs and symptoms that may occur before the appearance of clinically evident osteonecrosis include changes in the health of periodontal tissues, non-healing mucosal ulcers, loose teeth, and an unexplained soft tissue infection ${ }^{4}$.

Marx was the first to report osteonecrosis of the jaw due to administration of BPs in 2003. Since then, many studies have confirmed that BRONJ is an adverse side effect of BP therapy ${ }^{5,6)}$. In 2009, the American Association of Oral and Maxillofacial Surgeons (AAOMS) ${ }^{3}$ ) stated that the diagnosis of BRONJ is primarily determined by the presence of exposed necrotic bone in the oral cavity during an 8-week period, a history of current or previous BP intake, and the absence of irradiation of the jaws. In addition to bone exposure, common features are pain, swelling of soft tissues, ulceration, suppuration, and the development of sinus $\operatorname{tracts}^{7,8)}$. Moreover, many studies worldwide have reported an association between a range of serious dental diseases and the use of BPs. 


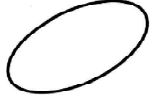

Type I

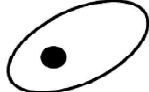

Type II

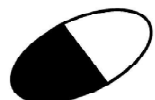

Type III

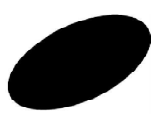

Type IV

Figure 1. Four signal patterns of the mandibular condyle bone marrow detected by MRI. Type I: Normal signal from the mandibular condyle bone marrow. Type II: A spot of abnormal signal from mandibular condyle bone marrow. Type III: Between a spot and half of the abnormal signal from mandibular condyle bone marrow. Type IV: one-half to completely abnormal signal from mandibular condyle bone marrow.

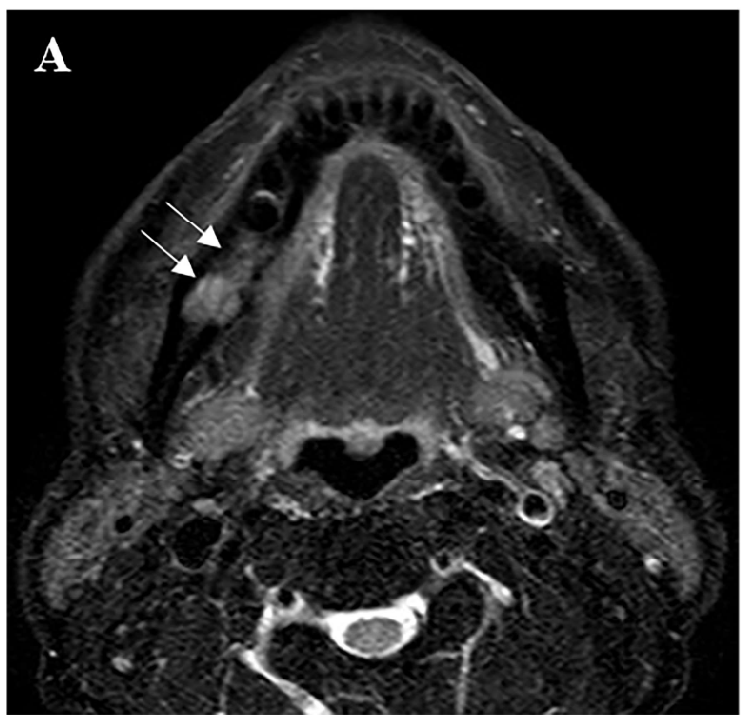

Figure 2. A 65-year-old woman with BRONJ. Axial STIR MRI shows inflammation of the mandible (A, arrow) and a normal signal from the mandibular condyle bone marrow (B, arrowhead).

BRONJ is usually diagnosed radiologically — e.g., panoramic radiography, dental cone beam computed tomography (CBCT), spiral CT. Osteolysis, osteosclerosis, thickening of the lamina dura, thickening of the periosteum, widening of the periodontal space, subperiosteal bone formation or sequestra, fracture, and radiologic evidence of sinusitis ${ }^{9)}$ are usually seen with BRONJ lesions. When clinically unexposed necrotic bone is seen, further examinations-e.g., bone scintigraphy, positron emission tomography, magnetic resonance imaging (MRI) - may help identify early areas of bone involvement ${ }^{10,11)}$. Imaging modalities such as radiography, scintigraphy, CT, and MRI are used for diagnostic bone marrow imaging. Among these modalities, MRI provides the best direct evaluation of all bone marrow components. Also, MRI is the only imaging technique that can visualize a bone marrow signal ${ }^{12-14)}$. Few studies, however, have evaluated BRONJ based on an abnormal signal detected by MRI in the bone marrow of the mandibular condyle.

The purpose of this study was to assess changes in the MRI signal of the mandibular condyle that could have been due to the presence of BRONJ. In particular, we focused on the relation of

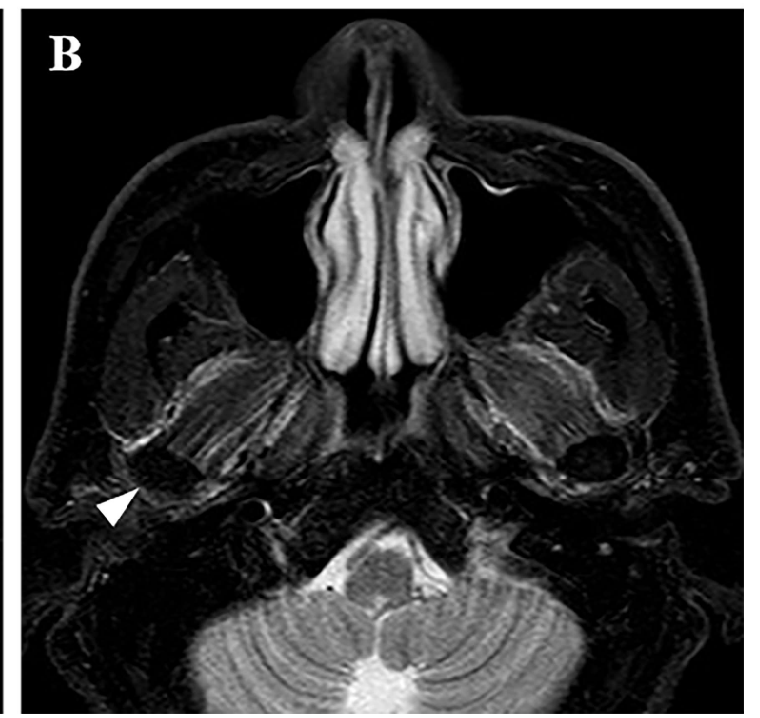

bone marrow signals of the mandibular condyle in BRONJ patients on the same side of the face that exhibited jaw symptoms versus on the side with no symptoms.

\section{Material and Methods}

\section{Study Design and Participants}

The university ethics committee approved this study (EC1512-009-1). Altogether, 28 patients (11 men, 17 women; 56 temporomandibular joints) were examined in this retrospective study. The patients (mean age $72.9 \pm 9.4$ years, range $48-88$ 
Naohisa Hirahara, et al.: Change in the MRI Signal of the Mandibular Condyle Due to BRONJ
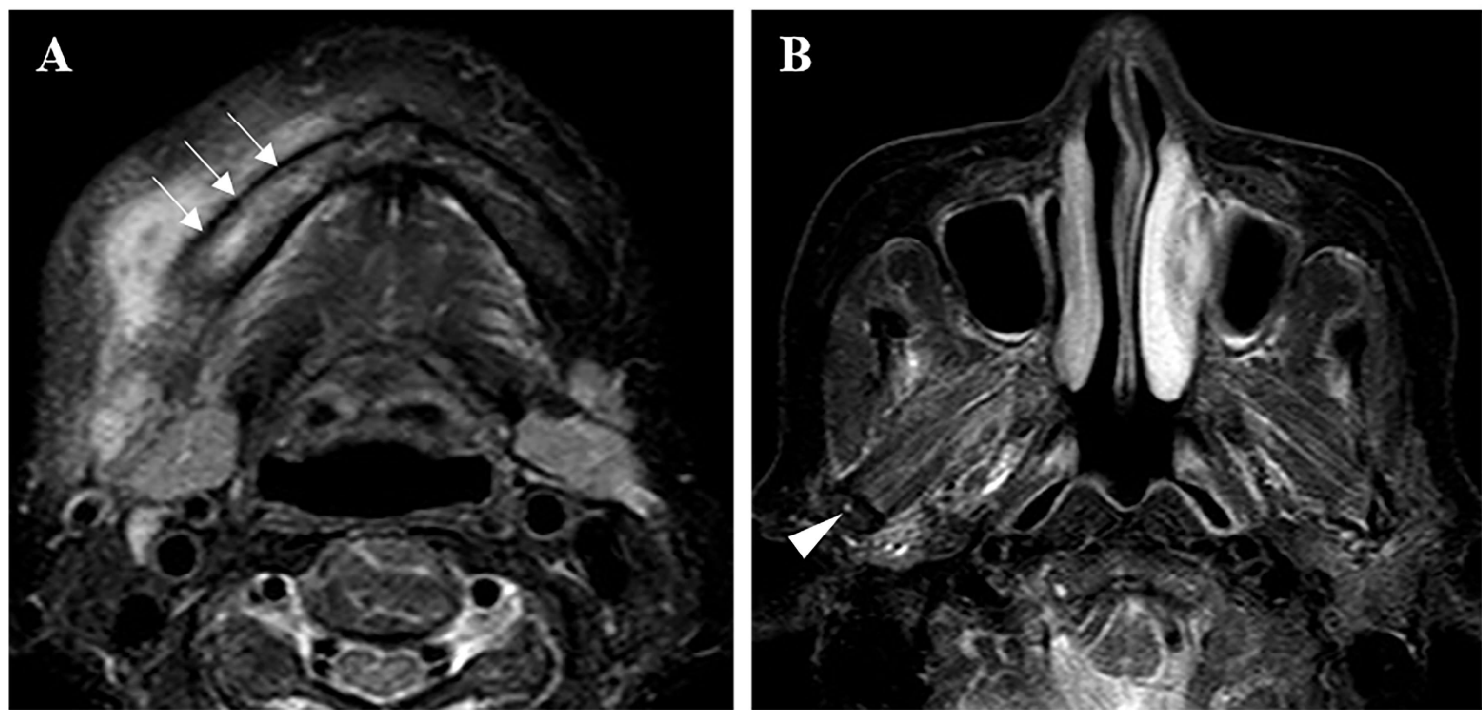

Figure 3. A 77-year-old woman with BRONJ. Axial STIR MRI shows inflammation of the mandible (A, arrow) and a spot of abnormal signal from the mandibular condyle bone marrow (B, arrowhead).
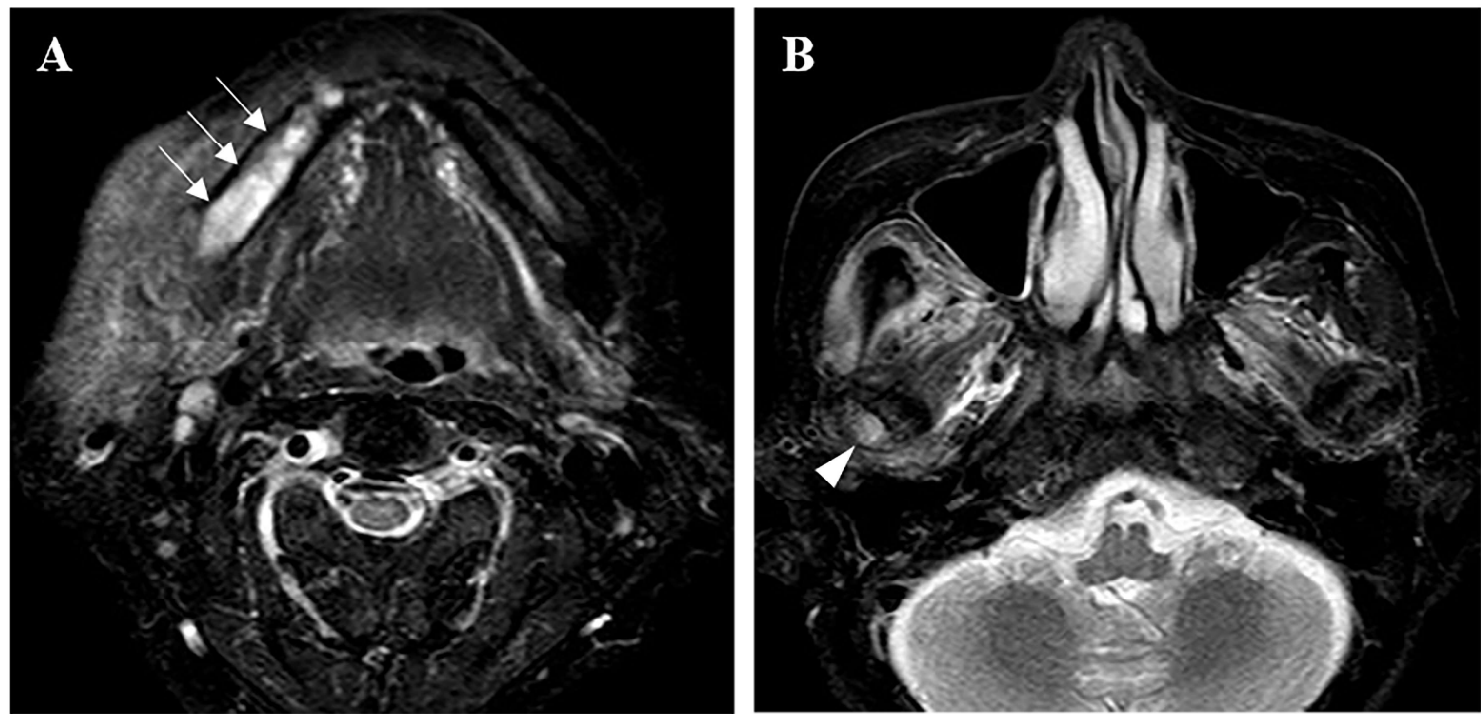

Figure 4. An 82-year-old woman with BRONJ. Axial STIR MRI shows inflammation of the mandible (A, arrow) and one-half abnormal signal from the mandibular condyle bone marrow (B, arrowhead)

mandibular condyle (Fig. 3)

- Type III: ranging from a spot to one-half abnormal bone marrow signal in the mandibular condyle (Fig. 4)

Type IV: between one-half and completely abnormal bone marrow signal in the mandibular condyle (Fig. 5)

These images were obtained using the axial plane of the maximum slice of the mandibular condyle. Any differences were resolved by forced consensus. These findings were then assessed in the BRONJ patients on the side of the face with symptoms and on the side with no symptoms. For cases of the signal crossing the midline or of symptoms on both sides, we assessed the images for both sides.

\section{Statistical Analysis}

Two oral radiologists independently evaluated the MRI scans for the presence or absence of the abnormal bone marrow signal from the mandibular condyle. Statistical analyses of the abnormal bone marrow signal regarding its relation to the side that displayed symptoms or the side with no symptoms was performed using the $\div^{2}$ test with Fisher's exact test (version 14.0; SPSS Japan, Tokyo, Japan). A value of $P<0.05$ was considered to indicate statistical significance. Another method of data analysis was the average value.

\section{Results}

This study assessed changes in the MRI signal from the mandibular condyle in BRONJ patients on the side of the face with jaw symptoms versus the MRI signal on the side with no 

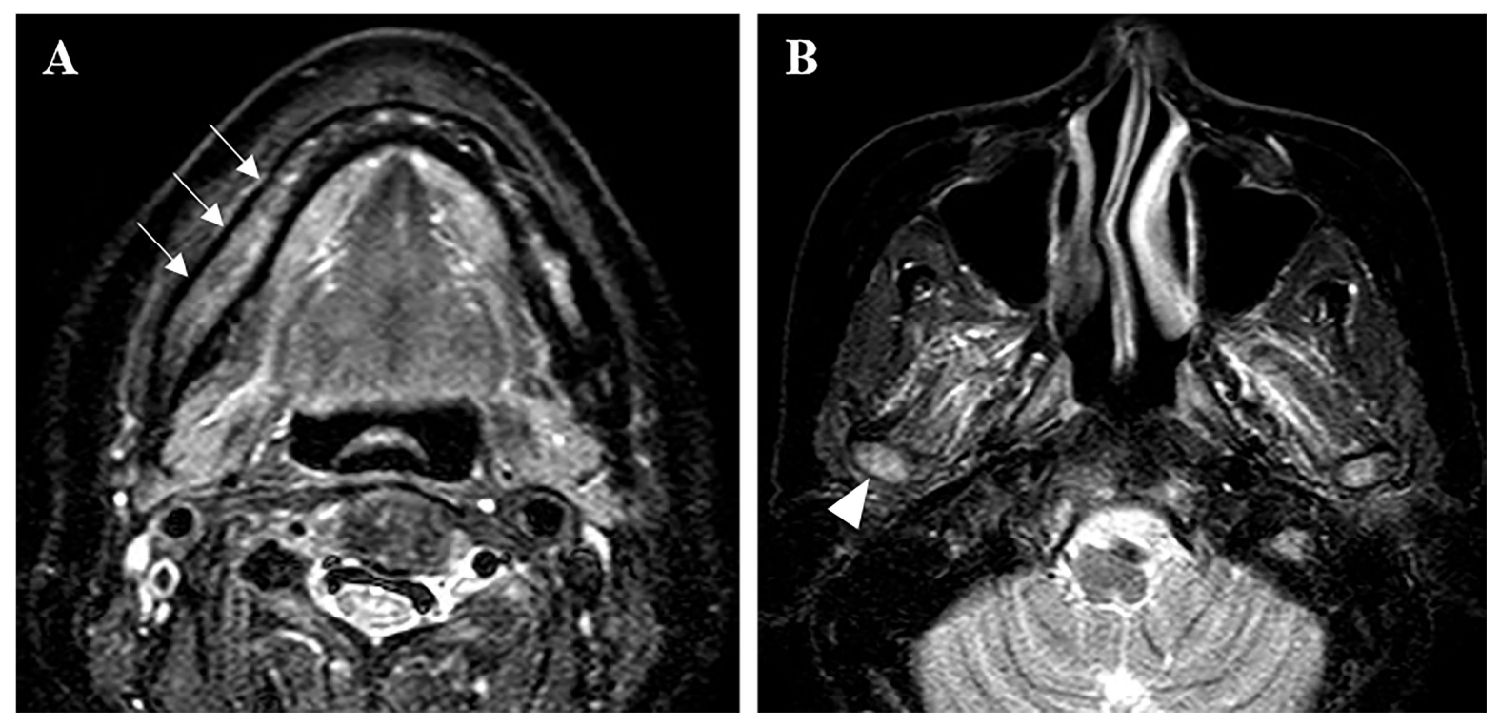

Figure 5. A 57-year-old woman with BRONJ. Axial STIR MRI shows inflammation of the mandible (A, arrow) and a completely abnormal signal of the mandibular condyle bone marrow (B, arrowhead).

Table 1. Abnormal MRI signal, according to the side of symptoms in BRONJ patients

\begin{tabular}{lll}
\hline $\begin{array}{l}\text { Abnormal signal on the } \\
\text { side of the jaw symptoms }\end{array}$ & \\
\hline & $24(80.0 \%)$ & - \\
Yes & $3(11.5 \%)$ & $<.05^{*}$ \\
\hline
\end{tabular}

*Significance of the difference between Yes vs. No.

symptoms. The results showed that $80.0 \%$ of the 24 mandibular condyles showed an abnormal bone marrow signal on the side of the face with symptoms. In contrast, only 3 mandibular condyles $(11.5 \%)$ of the BRONJ patients showed an abnormal bone marrow signal of the mandibular condyle on the side with no symptoms.

These data showed that an abnormal bone marrow signal of the mandibular condyle in BRONJ patients was significantly related to the appearance of symptoms $(P<0.05)$ (Table 1).

Among the 30 patients who had an abnormal bone marrow signal from the mandibular condyle on the same side as the symptoms, 5 (16.6\%) were type $1,16(53.3 \%)$ were type $2,3(10.0 \%)$ were type 3 , and $6(20.0 \%)$ were type 4 (Table 2 ).

\section{Discussion}

The MRI signal from the mandibular condyle on the same side as the symptoms had been changed by the presence of BRONJ in our study. Our study also showed a significant difference in the abnormal bone marrow signal of the mandibular condyle in these BRONJ patients in regard to the abnormal signal appearing on the same side as the symptoms or on the side with no symptoms. Although a correlation between the use of intravenously injected
Table 2. Type of mandibular condyle bone marrow signal in BRONJ patients with an abnormal signal and symptoms on the same side

\begin{tabular}{ll}
\hline Type & $\%$ \\
\hline I & 16.6 \\
II & 53.3 \\
III & 10.0 \\
IV & 20.0 \\
\hline
\end{tabular}

BPs and the development of BRONJ is now widely affirmed in the literature after being reported in 2003 by $\mathrm{Marx}^{6}$, the correlation between BRONJ and orally administered BPs is less clear or predictable ${ }^{2,15)}$.

The AAOMS described the diagnosis of BRONJ in 2009 and later suggested a staging system for BRONJ based on four stages $^{7,16)}$.

- Stage zero: represented by a nonexposed variant, in which other symptoms and signs are present (e.g., pain, formation of sinus tracts, radiologic markers) ${ }^{17}$.

- First stage: includes asymptomatic bone exposure.

- Second and third stages: include patients with exposed bone of various extents with other concomitant symptoms and signs that are mainly a result of secondary infection of the necrotic bone. The symptoms may include increased tooth mobility, formation of sinus tracts, suppuration and traumatic ulceration of oral mucosa adjacent to exposed bone, mandibular fracture, and/or cervical lymphadenopathy ${ }^{18)}$.

In the past, many studies worldwide have reported the diagnosis of BRONJ. Dental panoramic radiography and CT can be considered the most widely available imaging techniques for BRONJ evaluation ${ }^{19-21)}$, which explains why most of the selected studies have used those imaging modalities. Furthermore, they 
Naohisa Hirahara, et al.: Change in the MRI Signal of the Mandibular Condyle Due to BRONJ

usually detect dento-osseous changes related to this entity, including bone sclerosis, cortical surface irregularities, persistent extraction sockets, bone fragmentation (sequestration), and osteolysis $^{22)}$. MRI of BRONJ typically shows low signal intensity on T1-weighted images, medium-to-high signal intensity on T2weighted images or STIR images ${ }^{23)}$, and high signal intensity on contrast-enhanced T1-weighted images. In the advanced stage, the periphery of the necrotic bone is surrounded by osteomyelitic bone, for which T1-weighted images show low signal intensity and T2-weighted images and STIR images show high signal intensity. These signals indicate increased cellular components, osteogenesis, and vascular proliferation ${ }^{24,25)}$. Importantly, there is a report that a lesion that is clinically undetectable can be detected by MRI, suggesting that MRI could be useful for diagnosing earlystage BRONJ and that diagnostic imaging findings should be included in the definition of BRONJ ${ }^{26)}$.

The MRI features of normal marrow and the most current knowledge about marrow disorders are based on information provided by standard spin-echo pulse sequences with T1- and T2weighted images ${ }^{13)}$. The principal MRI examination techniques used for fat suppression are the STIR technique, shortening of inversion time (TI) during inversion recovery, and techniques making use of the frequency difference between the signals of water and fat, as represented by chemical shift techniques ${ }^{27)}$. Recently, MRI has become an important, noninvasive, imaging technique for bone marrow disorders. Clinical research with STIR provides improved assessment of bone marrow abnormalities ${ }^{28-}$ 30). The bone marrow in a normal adult consists of both red and yellow marrow ${ }^{31,32)}$. Red marrow gradually converts to yellow marrow with age, yielding changes in bone marrow patterns. Various diseases affect the bone marrow and can greatly affect its function ${ }^{13)}$. Therefore, evaluation of bone marrow is critical for the diagnosis, treatment, and prognosis of various diseases ${ }^{13)}$. Frequently, problems arise when determining whether an observed MRI marrow pattern is normal or abnormal ${ }^{12}$. Kaneda et al. described the MRI appearance of mandibular bone marrow at different ages. In the mandibular body, complete conversion from red marrow to yellow marrow occurs after 20 years of age. Therefore, normal mandibular bone marrow should have low signal intensity on STIR images ${ }^{31)}$. STIR imaging is useful for detecting mandibular osteomyelitis, identifying the extent of inflammation, investigating the spread of inflammation to soft tissues, and detecting postoperative recurrence of disease ${ }^{32)}$. In the present study, we used STIR for bone marrow evaluation because it is the principal MRI examination technique for fat suppression ${ }^{27)}$. Many studies worldwide have reported an association between BPs and a range of serious dental diseases ${ }^{33}$, 34). There have been few studies, however, that evaluated BRONJ with an abnormal bone marrow signal of the mandibular condyle using MRI. In this study, abnormal bone marrow signals of the mandibular condyle were observed in about $80.0 \%$ of BRONJ patients on the same side where symptoms had occurred in the mandibular condyle. To date, MRI findings of an abnormal bone marrow signal of the mandibular condyle in BRONJ patients have not been discussed in detail. We believe that our discovery could contribute to the early detection of BRONJ.

In conclusion, MRI signals of the mandibular condyle bone marrow on the same side that symptoms had occurred had been altered by the presence of BRONJ. This discovery suggests that MRI findings could be very useful clinically for detecting BRONJ in the mandibular condyle.

\section{Conflict of Interest}

The authors have declared that no COI exists.

\section{References}

1. Campisi G, Lo Russo L, Agrillo A, Vescovi P, Fusco V and Bedogni A. BRONJ expert panel recommendation of the italian societies for maxillofacial surgery (SICMF) and oral pathology and medicine (SIPMO) on bisphosphonate-related osteonecrosis of the jaw: risk assessment, preventive strategies and dental management. Ital J Maxillofac Surg 22: 103-124, 2011

2. Manzon L, Ettorre E, Viscogliosi G, Ippoliti S, Filiaci F, Ungari C, Fratto G and Agrillo A. Bisphosphonate therapy and osteonecrosis of the jaw complicated with a temporal abscess in an elderly woman with rheumatoid arthritis: a case report. Clin Interv Aging 9: 1409-1413, 2014

3. Ruggiero SL, Dodson TB, Assael LA, Landesberg R, Marx RE and Mebrotra B. American association of oral and maxillofacial surgeons position paper on bisphosphonaterelated osteonecrosis of the jaws $3 / 42009$ update. J Oral Maxillofac Surg 67: 2-12, 2009

4. Ficarra $G$ and Beninati F. Bisphosphonate-related osteonecrosis of the jaws: the point of view of the oral pathologist. Clin Cases Miner Bone Metab 4: 53-57, 2007

5. Nomura T, Shibahara T, Uchiyama T, Yamamoto N, Shibui T, Yakushiji T, Watanabe A, Muramatsu K, Ogane S, Murayama M, Sekine R, Nakata E and Fujimoto Y. Bisphosphonate-related osteonecrosis of jaws (BRONJ) in Japanese population: a case series of 13 patients at our clinic. Bull Tokyo Dent Coll 54: 117-125, 2013

6. Marx RE. Pamidronate (Aredia) and zoledronate (Zometa) induced avascular necrosis of the jaws: a growing epidemic. J Oral Maxillofac Surg 61: 1115-1117, 2003

7. Nicolatou-Galitis O, Papadopoulou E, Sarri T, Boziari P, Karayianni A, Kyrtsonis MC, Repousis P, Barbounis V and Migliorati CA. Osteonecrosis of the jaw in oncology patients treated with bisphosphonates: prospective experience of a dental oncology referral center. Oral Surg Oral Med Oral 
Pathol Oral Radiol Endod 112: 195-202, 2011

8. Paulo S, Abrantes AM, Laranjo M, Carvalho L, Serra A, Botelho MF and Ferreira MM. Bisphosphonate-related osteonecrosis of the jaw: Sspecificities. Oncol Rev 8: 254, 2014

9. Mast G, Otto S, Mücke T, Schreyer C, Bissinger O, Kolk A, Wolff KD, Ehrenfeld M, Stürzenbaum SR and Pautke C. Incidence of maxillary sinusitis and oro-antral fistulae in bisphosphonate-related osteonecrosis of the jaw. J Craniomaxillofac Surg 40: 568-571, 2012

10. O’Ryan FS, Khoury S, Liao W, Han MM, Hui RL, Baer D, Matin D, Liberty D and Lo JC. Intravenous bisphosphonaterelated osteonecrosis of the jaw: bone scintigraphy as an early indicator. J Oral Maxillofac Surg 67: 1363-1372, 2009

11. Longo F, Guida A, Aversa C, Pavone E, Di Costanzo G, Ramaglia L and Ionna F. Platelet rich plasma in the treatment of bisphosphonate-related osteonecrosis of the jaw: personal experience and review of the literature. Int J Dent 2014: 298945, 2014

12. Vande Berg BC, Malghem J, Lecouvet FE and Maldague B. Magnetic resonance imaging of the normal bone marrow. Skeletal Radiol 27: 471-483, 1998

13. Vogler JB 3rd and Murphy WA. Bone marrow imaging. Radiology 168: 679-693, 1988

14. Lieberman JM, Gardner CL, Motta AO and Schwartz RD. Prevalence of bone marrow signal abnormalities observed in the temporomandibular joint using magnetic resonance imaging. J Oral Maxillofac Surg 54: 434-439, discussion 439-440, 1996

15. Mavrokokki T, Cheng A, Stein B and Goss A. Nature and frequency of bisphosphonate-associated osteonecrosis of the jaws in Australia. J Oral Maxillofac Surg 65: 415-423, 2007

16. McLeod NM, Brennan PA and Ruggiero SL. Bisphosphonate osteonecrosis of the jaw: a historical and contemporary review. Surgeon 10: 36-42, 2012

17. Fedele S, Porter SR, D’Aiuto F, Aljohani S, Vescovi P, Manfredi M, Arduino PG, Broccoletti R, Musciotto A, Di Fede O, Lazarovici TS, Campisi G and Yarom N. Nonexposed variant of bisphosphonate-associated osteonecrosis of the jaw: a case series. Am J Med 123: 10601064,2010

18. Otto S, Schreyer C, Hafner S, Mast G, Ehrenfeld M, Stürzenbaum S and Pautke C. Bisphosphonate-related osteonecrosis of the jaws: Characteristics, risk factors, clinical features, localization and impact on oncological treatment. J Craniomaxillofac Surg 40: 303-309, 2012

19. Torres SR, Chen CS, Leroux BG, Lee PP, Hollender LG and Schubert MM. Fractal dimension evaluation of cone beam computed tomography in patients with bisphosphonateassociated osteonecrosis. Dentomaxillofac Radiol 40: 501-
505,2011

20. Rocha GC, Jaguar GC, Moreira CR, Neves EG, Fonseca FP and Pedreira EN. Radiographic evaluation of maxillofacial region in oncology patients treated with bisphosphonates. Oral Surg Oral Med Oral Pathol Oral Radiol Endod 114: S19-25, 2012

21. Stockmann P, Hinkmann FM, Lell MM, Fenner M, Vairaktaris E, Neukam FW and Nkenke E. Panoramic radiograph, computed tomography or magnetic resonance imaging: which imaging technique should be preferred in bisphosphonate-associated osteonecrosis of the jaw? A prospective clinical study. Clin Oral Investig 14: 311-317, 2010

22. Leite AF, dos Santos Ogata F, de Melo NS and de Souza Figueiredo PT. Imaging findings of bisphosphonate-related osteonecrosis of the jaws: a critical review of the quantitative studies. Int J Dent 2014: 1-11, 2014

23. Raje N, Woo SB, Hande K, Yap JT, Richardson PG, Vallet S, Treister N, Hideshima T, Sheehy N, Chhetri S, Connell B, Xie W, Tai YT, Szot-Barnes A, Tian M, Schlossman RL, Weller E, Munshi NC, Van Den Abbeele AD and Anderson KC. Clinical, radiographic, and biochemical characterization of multiple myeloma patients with osteonecrosis of the jaw. Clin Cancer Res 14: 2387-2395, 2008

24. Bedogni A, Blandamura S, Lokmic Z, Palumbo C, Ragazzo M, Ferrari F, Tregnaghi A, Pietrogrande F, Procopio O, Saia G, Ferretti M, Bedogni G, Chiarini L, Ferronato G, Ninfo V, Lo Russo L, Lo Muzio L and Nocini PF. Bisphosphonateassociated jawbone osteonecrosis: A correlation between imaging techniques and histopathology. Oral Surg Oral Med Oral Pathol Oral Radiol Endod 105: 358-364, 2008

25. Khosla S, Burr D, Cauley J, Dempster DW, Ebeling PR, Felsenberg D, Gagel RF, Gilsanz V, Guise T, Koka S, McCauley LK, McGowan J, McKee MD, Mohla S, Pendrys DG, Raisz LG, Ruggiero SL, Shafer DM, Shum L, Silverman SL, Van Poznak CH, Watts N, Woo SB and Shane E. Bisphosphonate-associated osteonecrosis of the jaw: report of a task force of the american society for bone and mineral research. J Bone Miner Res 22: 1479-1491, 2007

26. Garcia-Ferrer L, Bagán JV, Martinez-Sanjuan V, HernandezBazan S, Garcia R, Jimeìnez-Soriano Y and Hervas V. MRI of mandibular osteonecrosis secondary to bisphosphonates. AJR Am J Roentgenol 190: 949-955, 2008

27. Lee K, Kaneda T, Mori S, Minami M, Motohashi J and Yamashiro M. Magnetic resonance imaging of normal and osteomyelitis in the mandible: assessment of short inversion time inversion recovery sequence. Oral Surg Oral Med Oral Pathol Radiol Endod 96: 499-507, 2003

28. Zanetti M, Bruder E, Romero J and Hodler J. Bone marrow edema pattern in osteoarthritic knees: correlation between 
Naohisa Hirahara, et al.: Change in the MRI Signal of the Mandibular Condyle Due to BRONJ

MR imaging and histologic findings. Radiology 215: 835840,2000

29. Hayes CW, Conway WF and Daniel WW. MR imaging of bone marrow edema pattern: transient osteoporosis, transient bone marrow edema syndrome, or osteonecrosis. Radiographics 13: 1001-1011, 1993

30. Reinus WR, Fischer KC and Ritter JH. Painful transient tibial edema. Radiology 192: 195-199, 1994

31. Kaneda T, Minami M, Ozawa K, Akimoto Y, Okada H, Yamamoto H, Suzuki H and Sasaki Y. Magnetic resonance appearance of bone marrow in the mandible at different ages. Oral Surg Oral Med Oral Pathol Radiol Endod 82: 229233, 1996
32. Kaneda T, Minami M, Ozawa K, Akimoto Y, Utsunomiya T, Yamamoto H, Suzuki H and Sasaki Y. Magnetic resonance imaging of osteomyelitis in the mandible: comparative study with other radiologic modalities. Oral Surg Oral Med Oral Pathol Radiol Endod 79: 634-640, 1995

33. Phal PM, Myall RW, Assael LA and Weissman JL. Imaging findings of bisphosphonate-associated osteonecrosis of the jaws. AJNR Am J Neuroradiol 28: 1139-1145, 2007

34. Marx RE, Sawatari Y, Fortin M and Broumand V. Bisphosphonate-induced exposed bone (osteonecrosis/ osteopetrosis) of the jaws: Rrisk factors, recognition, prevention, and treatment. J Oral Maxillofac Surg 63: 15671575,2005 
J.Hard Tissue Biology Vol. 26(2): 161- 168, 2017 